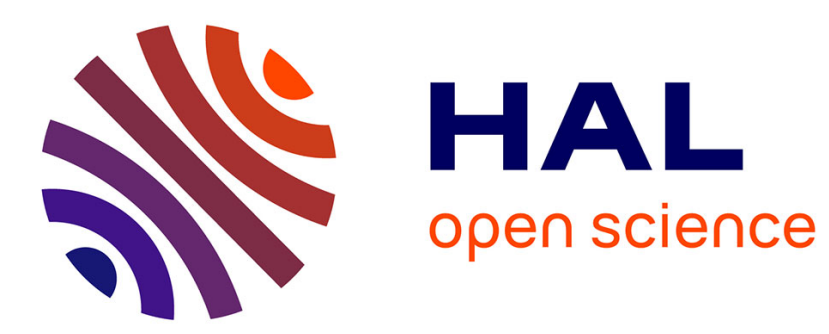

\title{
Weakened conditions of admissibility of surface forces applied to linearly elastic membrane shells
}

Robert Luce, Cécile Poutous, Jean-Marie Thomas

\section{To cite this version:}

Robert Luce, Cécile Poutous, Jean-Marie Thomas. Weakened conditions of admissibility of surface forces applied to linearly elastic membrane shells. Analysis and Applications, 2008, 6 (3), pp.247-267. inria-00341897

\section{HAL Id: inria-00341897 \\ https://hal.inria.fr/inria-00341897}

Submitted on 26 Nov 2008

HAL is a multi-disciplinary open access archive for the deposit and dissemination of scientific research documents, whether they are published or not. The documents may come from teaching and research institutions in France or abroad, or from public or private research centers.
L'archive ouverte pluridisciplinaire HAL, est destinée au dépôt et à la diffusion de documents scientifiques de niveau recherche, publiés ou non, émanant des établissements d'enseignement et de recherche français ou étrangers, des laboratoires publics ou privés. 


\title{
Weakened conditions of admissibility of surface forces applied to linearly elastic membrane shells
}

\author{
Robert Luce, Cécile Poutous, Jean-Marie Thomas \\ robert.luce@univ-pau.fr, cecile.poutous@univ-pau.fr, jean-marie.thomas@univ-pau.fr \\ Laboratoire de Mathématiques Appliquées, UMR 5142, Université de Pau et des Pays de l'Adour, BP 1155, 64013 Pau Cedex, France
}

\begin{abstract}
We consider a family of linearly elastic shells of the first kind (as defined in Ciarlet [2]), also known as non inhibited pure bending shells (Sanchez-Hubert and Sanchez-Palencia [7]). This family is indexed by the half-thickness $\varepsilon$. When $\varepsilon$ approaches zero, the averages across the thickness of the shell of the covariant components of the displacement of the points of the shell converge strongly towards the solution of a "2D generalized membrane shell problem" provided the applied forces satisfy admissibility conditions (Ciarlet and Lods [3], Chapelle and Bathe [1]). The identification of the admissible applied forces usually requires delicate analysis.

In the first part of this paper we simplify the general admissibility conditions when applied forces $\mathbf{h}$ are surface forces only, and obtain conditions that no longer depend on $\varepsilon$ (Luce, Poutous and Thomas [5]) : find $h^{\alpha \beta}=h^{\beta \alpha}$ in $L^{2}(\omega)$ such that for all $\eta=\left(\eta_{i}\right)$ in $\mathbf{V}(\omega), \int_{\omega} h^{i} \eta_{i} d \omega=\int_{\omega} h^{\alpha \beta} \gamma_{\alpha \beta}(\eta) d \omega$ where $\omega$ is a domain of $\mathbb{R}^{2}, \theta$ is in $C^{3}\left(\bar{\omega}, \mathbb{R}^{3}\right)$ and $S=\theta(\bar{\omega})$ is the middle surface of the shells, where $\left(\gamma_{\alpha \beta}(\eta)\right)$ is the linearized strain tensor of $S$ and $\mathbf{V}(\omega)=\left\{\eta \in \mathbf{H}^{1}(\omega), \eta=\mathbf{0}\right.$ on $\left.\gamma_{0}\right\}$, the shells being clamped along $\Gamma_{0}=\theta\left(\gamma_{0}\right)$.

In the second part, since the simplified admissibility formulation does not allow to conclude directly to the existence of $h^{\alpha \beta}$, we seek sufficient conditions on $\mathbf{h}$ for $h^{\alpha \beta}$ to exist in $L^{2}(\omega)$. In order to get them, we impose more regularity to $h^{\alpha \beta}$ and boundary conditions. Under these assumptions, we can obtain from the weak formulation a system of PDE with $h^{\alpha \beta}$ as unknowns. The existence of solutions depends both on the geometry of the shell and on the choice of $\mathbf{h}$. We carry through the study of four representative geometries of shells and identify in each case a special admissibility functional space for $\mathbf{h}$.
\end{abstract}

\section{Introduction and notations}

In this paper, greek indices take their values in $\{1,2\}$, whereas latin indices belong to $\{1,2,3\}$ and the repeated index summation convention is used.

Let us first consider the "2D" ill-posed scaled variational problem

$$
\mathcal{P}(\omega):\left\{\begin{array}{l}
\zeta \in \mathbf{V}(\omega):=\left\{\eta=\left(\eta_{i}\right) \in \mathbf{H}^{1}(\omega) ; \eta=\mathbf{0} \text { on } \gamma_{0}\right\}, \forall \eta \in \mathbf{V}(\omega) \\
\int_{\omega} a^{\alpha \beta \sigma \tau} \gamma_{\sigma \tau}(\zeta) \gamma_{\alpha \beta}(\eta) \sqrt{a} d y=\int_{\omega} h^{i} v_{i} \sqrt{a} d y
\end{array}\right.
$$

where the bilinear form is not coercive on $\mathbf{V}(\omega)$, the surface functions $h^{i} \in L^{2}(\omega)$ are independent of $\varepsilon$, $\omega$ is a domain in $\mathbb{R}^{2}$ (open, bounded, connected subset with a Lipschitz-continuous boundary, the set $\omega$ being locally on one side of its boundary), $\theta: \bar{\omega} \longrightarrow \mathbb{R}^{3}\left(\theta \in C^{3}\left(\bar{\omega} ; \mathbb{R}^{3}\right)\right)$ is an injective mapping such that the two vectors $\mathbf{a}_{\alpha}:=\partial_{\alpha} \theta(y)$ are linearly independent at each point $y \in \bar{\omega}$, where $\mathbf{a}_{3}:=\frac{\mathbf{a}_{1} \wedge \mathbf{a}_{2}}{\left|\mathbf{a}_{1} \wedge \mathbf{a}_{2}\right|}$, and $a_{\alpha \beta}:=\mathbf{a}_{\alpha} \cdot \mathbf{a}_{\beta}$ denote the covariant components of the metric tensor of $S:=\theta(\bar{\omega})$, and $a:=\operatorname{det}\left(\left(a_{\alpha \beta}\right)_{\alpha \beta}\right), a^{\alpha \beta}$ denote the contravariant components of the metric tensor of 
$S:=\theta(\bar{\omega})$, where $a^{\alpha \beta \sigma \tau}$ which denote the contravariant components of the scaled 2D elasticity tensor are defined by

$$
a^{\alpha \beta \sigma \tau}:=\frac{4 \lambda \mu}{\lambda+2 \mu} a^{\alpha \beta} a^{\sigma \tau}+2 \mu\left(a^{\alpha \sigma} a^{\beta \tau}+a^{\alpha \tau} a^{\beta \sigma}\right) \text { with } \lambda>0, \mu>0
$$

where $\Gamma_{\alpha \beta}^{\sigma}$ are the surface Christoffel symbols i.e. $\Gamma_{\alpha \beta}^{\sigma}:=\mathbf{a}^{\sigma} \cdot \partial_{\alpha} \mathbf{a}_{\beta}$ with $\mathbf{a}^{i} \cdot \mathbf{a}_{j}=\delta_{i j}$, and where, for any vector field $\eta=\left(\eta_{i}\right) \in \mathbf{H}^{1}(\omega)$, the covariant components of the $2 \mathrm{D}$ linearized change of metric tensor $\gamma_{\alpha \beta}(\eta) \in L^{2}(\omega)$ are defined by

$$
\gamma_{\alpha \beta}(\eta):=\frac{1}{2}\left(\partial_{\beta} \eta_{\alpha}+\partial_{\alpha} \eta_{\beta}\right)-\Gamma_{\alpha \beta}^{\sigma} \eta_{\sigma}-b_{\alpha \beta} \eta_{3} \text { with } b_{\alpha \beta}:=\mathbf{a}_{3} \cdot \partial_{\alpha} \mathbf{a}_{\beta}
$$

Let us also consider the 3D scaled variational problem

$$
\mathcal{P}(\varepsilon ; \Omega):\left\{\begin{array}{l}
\mathbf{u}(\varepsilon) \in \mathbf{V}(\Omega):=\left\{\mathbf{v}=\left(v_{i}\right) \in \mathbf{H}^{1}(\Omega) ; \mathbf{v}=\mathbf{0} \text { on } \Gamma_{0}:=\gamma_{0} \times[-1,1]\right\}, \forall \mathbf{v} \in \mathbf{V}(\Omega) \\
\int_{\Omega} A^{i j k l}(\varepsilon) e_{k \| l}(\varepsilon ; \mathbf{u}(\varepsilon)) e_{i \| j}(\varepsilon ; \mathbf{v}) \sqrt{g(\varepsilon)} d x=\int_{\Gamma^{+} \cup \Gamma^{-}} h^{i \pm} v_{i} \sqrt{g(\varepsilon)} d \Gamma
\end{array}\right.
$$

where the functions $h^{i \pm} \in L^{2}\left(\Gamma^{+} \cup \Gamma^{-}\right)$are independent of $\left.\varepsilon, \Omega:=\omega \times\right]-1,1\left[, \Gamma^{+}:=\omega \times\{1\}, \Gamma^{-}:=\omega \times\{-1\}\right.$, and $\left.\Omega_{\varepsilon}:=\omega \times\right]-\varepsilon, \varepsilon\left[, \boldsymbol{\Theta}: \bar{\Omega}_{\varepsilon} \longrightarrow \mathbb{R}^{3}\right.$ is the canonical extension of $\theta$ and thus verifies $\boldsymbol{\Theta}\left(y, x_{3}\right):=\theta(y)+x_{3} a_{3}$ and $\operatorname{det}\left(\mathbf{g}_{1}, \mathbf{g}_{2}, \mathbf{g}_{3}\right)>0$ (where $\mathbf{g}_{i}:=\partial_{i} \boldsymbol{\Theta}$ ), where, for any vector field $\mathbf{v}=\left(v_{i}\right) \in \mathbf{H}^{1}(\Omega)$, the scaled linearized strains $e_{i \| j}(\varepsilon ; \mathbf{v})=e_{j \| i}(\varepsilon ; \mathbf{v}) \in \mathbf{L}^{2}(\Omega)$ are defined by

$$
\begin{array}{r}
e_{\alpha \| \beta}(\varepsilon ; \mathbf{v}):=\frac{1}{2}\left(\partial_{\beta} v_{\alpha}+\partial_{\alpha} v_{\beta}\right)-\Gamma_{\alpha \beta}^{p}(\varepsilon) v_{p} \\
e_{\alpha \| 3}(\varepsilon ; \mathbf{v}):=\frac{1}{2}\left(\frac{1}{\varepsilon} \partial_{3} v_{\alpha}+\partial_{\alpha} v_{3}\right)-\Gamma_{\alpha 3}^{\sigma}(\varepsilon) v_{\sigma}, e_{3 \| 3}(\varepsilon ; \mathbf{v}):=\frac{1}{\varepsilon} \partial_{3} v_{3}
\end{array}
$$

with $\Gamma_{i j}^{p}(\varepsilon): \bar{\Omega} \longrightarrow \mathbb{R}$ being the scaled 3D Christoffel symbols i.e.

$$
\Gamma_{i j}^{p}(\varepsilon)\left(x_{1}, x_{2}, x_{3}\right):=\Gamma_{i j}^{\varepsilon, p}\left(x_{1}, x_{2}, \varepsilon x_{3}\right) \text { and } \Gamma_{i j}^{\varepsilon, p}:=\mathbf{g}^{p} \cdot \partial_{i} \mathbf{g}_{j} \text { with } \mathbf{g}^{i} \cdot \mathbf{g}_{j}=\delta_{i j},
$$

with also, $g(\varepsilon): \bar{\Omega} \longrightarrow \mathbb{R}$ being the scaled function of $g^{\varepsilon}:=\operatorname{det}\left(\mathbf{g}_{i} \cdot \mathbf{g}_{j}\right)$, i.e $g(\varepsilon)\left(x_{1}, x_{2}, x_{3}\right):=g^{\varepsilon}\left(x_{1}, x_{2}, \varepsilon x_{3}\right)$, and where, at last, the contravariant components $A^{i j k l}(\varepsilon): \bar{\Omega} \longrightarrow \mathbb{R}$ of the scaled $3 \mathrm{D}$ elasticity tensor satisfy

$$
A^{i j k l}(\varepsilon)=A^{j i k l}(\varepsilon)=A^{k l i j}(\varepsilon), A^{i j k l}(\varepsilon)=A^{i j k l}(0)+O(\varepsilon) \text { and } A^{\alpha \beta \sigma 3}(\varepsilon)=A^{\alpha 333}(\varepsilon)=0,
$$

where the order symbol is meant with respect to the norm $\|w\|_{0, \infty, \bar{\Omega}}:=\sup \{|w(x)|, x \in \bar{\Omega}\}$ and

$$
\begin{aligned}
A^{\alpha \beta \sigma \tau}(0) & :=\lambda a^{\alpha \beta} a^{\sigma \tau}+\mu\left(a^{\alpha \sigma} a^{\beta \tau}+a^{\alpha \tau} a^{\beta \sigma}\right), A^{\alpha \beta 33}(0):=\lambda a^{\alpha \beta}, A^{\alpha 3 \sigma 3}(0):=\mu a^{\alpha \sigma} \\
A^{3333}(0) & :=\lambda+2 \mu, A^{\alpha \beta \sigma 3}(0)=A^{\alpha 333}(0):=0 .
\end{aligned}
$$

Let us now assume that the semi norm $|\cdot|_{\omega}^{M}$ defined by $|\eta|_{\omega}^{M}:=\left(\sum_{\alpha, \beta}\left|\gamma_{\alpha \beta}(\eta)\right|_{0, \omega}^{2}\right)^{1 / 2}$ is a norm over the space $\mathbf{V}(\omega)$ which is not equivalent to the norm $\|\cdot\|_{1, \omega}$ (Slicaru [9]) and let $\mathbf{V}_{M}^{\#}(\omega)$ be the completion of $\mathbf{V}(\omega)$ with respect to $|\cdot|_{\omega}^{M}$. Let $|\cdot|_{\Omega}^{M}$ be the norm over $\mathbf{V}(\Omega)$ defined by

$$
|\mathbf{v}|_{\Omega}^{M}=\left\{\left|\partial_{3} \mathbf{v}\right|_{0, \Omega}^{2}+\left(|\overline{\mathbf{v}}|_{\omega}^{M}\right)^{2}\right\}^{1 / 2} \text { where } \overline{\mathbf{v}}:=\frac{1}{2} \int_{-1}^{1} \mathbf{v} d x_{3}
$$

and let $\mathbf{V}_{M}^{\#}(\Omega)$ be the completion of $\mathbf{V}(\Omega)$ with respect to $|\cdot|_{\Omega}^{M}$.

Let $B_{M}(\zeta, \eta):=\int_{\omega} a^{\alpha \beta \sigma \tau} \gamma_{\sigma \tau}(\zeta) \gamma_{\alpha \beta}(\eta) \sqrt{a} d y$ and $L_{M}(\eta):=\int_{\omega} h^{i} \eta_{i} \sqrt{a} d y$ and let $B_{M}^{\#}$ and $L_{M}^{\#}$ denote the unique continuous extensions from $\mathbf{V}(\omega)$ to $\mathbf{V}_{M}^{\#}(\omega)$ of the bilinear form $B_{M}$ and the linear form $L_{M}$.

Under all these assumptions, Ph. Ciarlet and V. Lods proved that 
Theorem 1 There exist $\mathbf{u}$ in $\mathbf{V}_{M}^{\#}(\Omega)$ and $\zeta$ in $\mathbf{V}_{M}^{\#}(\omega)$ such that

$$
\mathbf{u}(\varepsilon) \longrightarrow \mathbf{u} \text { in } \mathbf{V}_{M}^{\#}(\Omega) \text { as } \varepsilon \rightarrow 0 \text { and } \overline{\mathbf{u}(\varepsilon)} \longrightarrow \zeta \text { in } \mathbf{V}_{M}^{\#}(\omega) \text { as } \varepsilon \rightarrow 0 .
$$

and the limit $\zeta$ satisfies the scaled $2 D$ variational problem of a linearly elastic generalized membrane shell of the first kind

$$
\mathcal{P}_{\mathcal{M}}^{\#}(\omega):\left\{\begin{array}{l}
\zeta \in \mathbf{V}_{M}^{\#}(\omega), \forall \eta \in \mathbf{V}_{M}^{\#}(\omega) \\
B_{M}^{\#}(\zeta, \eta)=L_{M}^{\#}(\eta)
\end{array}\right.
$$

if the density of surface force $\boldsymbol{h}$ is admissible, that is, if there exist for each $\varepsilon, 0<\varepsilon<\varepsilon_{0}$, functions $F^{i j}(\varepsilon)=$ $F^{j i}(\varepsilon) \in L^{2}(\Omega)$ and there exist functions $F^{i j}=F^{j i} \in L^{2}(\Omega)$ such that $F^{i j}(\varepsilon) \longrightarrow F^{i j}$ in $L^{2}(\Omega)$ as $\varepsilon \rightarrow 0$ and

$$
\int_{\Gamma^{+} \cup \Gamma^{-}} h^{i \pm} v_{i} \sqrt{g(\varepsilon)} d \Gamma=\int_{\Omega} F^{i j}(\varepsilon) e_{i \| j}(\varepsilon ; \mathbf{v}) \sqrt{g(\varepsilon)} d x \text { for all } 0<\varepsilon<\varepsilon_{0} \text { and for all } \mathbf{v} \in \mathbf{V}(\Omega) \text {. }
$$

Remark 1 Because of the previous strong convergency results, it seems natural to carry on with the study of admissibility conditions. But it is not the only option, an alternative is to study the behaviour of the solution $\mathbf{u}(\varepsilon)$ when the forces are not admissible. V. Lods and C. Mardare have proved in [4] that, provided the shell is totally clamped, the solutions $\mathbf{u}(\varepsilon)$ strongly converge, in the energy norm, towards the displacement given by Koiter or Naghdi's models.

\section{Main results}

In what follows we assume that all the assumptions above are satisfied. Let us now simplify the second part of the previous theorem and prove that

Theorem 2 There exist $\mathbf{u}$ in $\mathbf{V}_{M}^{\#}(\Omega)$ and $\zeta$ in $\mathbf{V}_{M}^{\#}(\omega)$ such that

$$
\mathbf{u}(\varepsilon) \longrightarrow \mathbf{u} \text { in } \mathbf{V}_{M}^{\#}(\Omega) \text { as } \varepsilon \rightarrow 0 \text { and } \overline{\mathbf{u}(\varepsilon)} \longrightarrow \zeta \text { in } \mathbf{V}_{M}^{\#}(\omega) \text { as } \varepsilon \rightarrow 0
$$

and the limit $\zeta$ satisfies the scaled $2 D$ variational problem $\mathcal{P}_{\mathcal{M}}^{\#}(\omega)$ if there exist functions $h^{\alpha \beta}=h^{\beta \alpha} \in L^{2}(\omega)$ such that the density of surface force $\boldsymbol{h}$ satisfies :

$$
\int_{\omega} h^{i} \eta_{i} \sqrt{a} d y=\int_{\omega} h^{\alpha \beta} \gamma_{\alpha \beta}(\eta) \sqrt{a} d y \text { for all } \eta \in \mathbf{V}(\omega) .
$$

The proof is given for a density applied on the upper surface so that we can identify $h^{i \pm}$ with $h^{i}$. The general case is then proved by linearity. In Ciarlet and Lods [3], the proof is divided in ten parts. To prove our theorem, we keep the same pattern of proof. But we only have to change the proof of parts $(i i),(i i i),(v)$ and (vii) since these are the parts concerned with the admissibility of the forces. The proof of the other parts remains unchanged. For a better understanding of the whole proof, we remind them and use their results when required.

Before proving Theorem 2, let us first remind two usefull propositions already proved in Ciarlet [2].

Proposition 1 We have the following $3 D$ Inequality of Korn's type : there exist constants $C>0$ and $\varepsilon_{0}>0$ such that

$$
\|\mathbf{v}\|_{1, \Omega} \leq \frac{C}{\varepsilon}\left\{\sum_{i, j}\left\|e_{i \| j}(\varepsilon ; \mathbf{v})\right\|_{0, \Omega}^{2}\right\}^{1 / 2} \text { for all } \mathbf{v} \in \mathbf{V}(\Omega) \text { and all } 0<\varepsilon<\varepsilon_{0} .
$$

Proposition 2 If $w \in L^{2}(\Omega)$ satisfies

$$
\int_{\Omega} w \partial_{3} v d x=0 \text { for all } v \in H^{1}(\Omega) \text { that vanish on } \Gamma_{0}, \text { then } w=0 \text {. }
$$


We now prove two preliminary results that will be used in the proof of Theorem $\mathbf{2}$.

Lemma 1 For $v \in H^{1}(\Omega)$, let $v_{\mid \Gamma^{+}}$denote the trace of $v$ on $\Gamma^{+}$and $\bar{v}$ denote the mean value of $v$ in the thickness. Then we have

$$
v_{\mid \Gamma^{+}}=\bar{v}+\frac{1}{2} \int_{-1}^{1}\left(1+x_{3}\right) \partial_{3} v d x_{3} .
$$

Proof. The result is obtained after the following integration by parts:

$$
\begin{aligned}
\bar{v} & =\frac{1}{2} \int_{-1}^{1} v d x_{3}=\frac{1}{2}\left(\int_{-1}^{1} \partial_{3}\left(\left(1+x_{3}\right) v\right) d x_{3}-\int_{-1}^{1}\left(1+x_{3}\right) \partial_{3} v d x_{3}\right) \\
& =\frac{1}{2}\left((1+1) v_{\mid \Gamma^{+}}-(1-1) v_{\mid \Gamma^{-}}-\int_{-1}^{1}\left(1+x_{3}\right) \partial_{3} v d x_{3}\right) .
\end{aligned}
$$

Lemma 2 There exist constants $c>0, \varepsilon_{0}>0$ and a function $G\left(\varepsilon, x_{1}, x_{2}, x_{3}\right)$ such that for all $0<\varepsilon<\varepsilon_{0}$,

$$
\sqrt{g(\varepsilon)}=\sqrt{a}+\varepsilon G \text { with }\|G\|_{0, \infty, \bar{\Omega}} \leq c
$$

Proof. In Ciarlet [2], p156, it is proved that $g_{\alpha \beta}(\varepsilon)=a_{\alpha \beta}-2 \varepsilon x_{3} b_{\alpha \beta}+O\left(\varepsilon^{2}\right)$. Then, since $g(\varepsilon)=\operatorname{det}\left(\left(g_{i j}(\varepsilon)\right)_{i, j}\right)$ and $a=\operatorname{det}\left(\left(a_{\alpha \beta}\right)_{\alpha \beta}\right)$, we have the result by using a first order Taylor development.

We can now give the proof of Theorem 2.

Proof.

Part (i): (no proof) There exist constants $c_{0}>0$ and $\varepsilon_{0}>0$ such that

$$
|\mathbf{v}|_{\Omega}^{M} \leq c_{0}\left\{\sum_{i, j}\left\|e_{i \| j}(\varepsilon ; \mathbf{v})\right\|_{0, \Omega}^{2}\right\}^{1 / 2} \text { for all } \mathbf{v} \in \mathbf{V}(\Omega) \text { and all } 0<\varepsilon<\varepsilon_{0} .
$$

Part (ii): (partial proof) There is a subsequence, still denoted $(\mathbf{u}(\varepsilon))_{\varepsilon>0}$ for convenience, and there exist $\mathbf{u} \in \mathbf{V}_{M}^{\#}(\Omega)$, $\mathbf{u}^{-1}=\left(u_{i}^{-1}\right) \in \mathbf{V}(\Omega), e_{i \| j} \in L^{2}(\Omega)$, and $\zeta \in \mathbf{V}_{M}^{\#}(\omega)$ such that

$$
\begin{array}{llll}
\mathbf{u}(\varepsilon) & \rightarrow & \mathbf{u} & \text { in } \mathbf{V}_{M}^{\#}(\Omega), \\
\varepsilon \mathbf{u}(\varepsilon) & \rightarrow & \mathbf{u}^{-1} & \text { in } \mathbf{H}^{1}(\Omega), \\
e_{i \| j}(\varepsilon) & \rightarrow & e_{i \| j} & \text { in } L^{2}(\Omega), \\
\varepsilon e_{3 \| 3}(\varepsilon) & \longrightarrow & e_{3 \| 3} & \text { in } L^{2}(\Omega), \\
\mathbf{u}(\varepsilon) & \rightarrow & \zeta & \text { in } \mathbf{V}_{M}^{\#}(\omega) \text { as } \varepsilon \rightarrow 0
\end{array}
$$

In order to prove that, it is sufficient to prove that there exist constants $c>0$ and $\varepsilon_{1}>0$ such that for all $0<\varepsilon<\varepsilon_{1}$,

$$
\left|\int_{\Gamma^{+}} h^{i+} u_{i}(\varepsilon) \sqrt{g(\varepsilon)} d \Gamma\right| \leq c\left\{\sum_{i, j}\left\|e_{i \| j}(\varepsilon)\right\|_{0, \Omega}^{2}\right\}^{1 / 2} .
$$

From (8) we know that

$$
\int_{\Gamma^{+}} h^{i+} u_{i}(\varepsilon) \sqrt{g(\varepsilon)} d \Gamma=\int_{\Gamma^{+}} h^{i+} u_{i}(\varepsilon) \sqrt{a} d \Gamma+\int_{\Gamma^{+}} h^{i+} u_{i}(\varepsilon) \varepsilon G d \Gamma .
$$

Then, with the help of (4) and (7), we can write the first integral of the rigthside of (10) this way:

$$
\int_{\Gamma^{+}} h^{i+} u_{i}(\varepsilon) \sqrt{a} d \Gamma=\int_{\omega} h^{\alpha \beta} \gamma_{\alpha \beta}(\overline{\mathbf{u}(\varepsilon)}) \sqrt{a} d y+\frac{1}{2} \int_{\Omega}\left(1+x_{3}\right) \mathbf{h} \partial_{3} \mathbf{u}(\varepsilon) \sqrt{a} d x
$$


Hence, applying Cauchy-Schwarz inequality first, using the definition of $|\mathbf{u}(\varepsilon)|_{\Omega}^{M}$ afterwards, and lastly using (9) we have the following inequalities

$$
\begin{aligned}
\left|\int_{\Gamma^{+}} h^{i+} u_{i}(\varepsilon) \sqrt{a} d \Gamma\right| & \leq\left\|h^{\alpha \beta} \sqrt{a}\right\|_{0, \omega}\left\|\gamma_{\alpha \beta}(\overline{\mathbf{u}(\varepsilon)})\right\|_{0, \omega}+\frac{1}{2}\left\|\left(1+x_{3}\right) \mathbf{h} \sqrt{a}\right\|_{0, \Omega}\left\|\partial_{3} \mathbf{u}(\varepsilon)\right\|_{0, \Omega} \\
& \leq c|\mathbf{u}(\varepsilon)|_{\Omega}^{M} \leq c\left\{\sum_{i, j}\left\|e_{i \| j}(\varepsilon)\right\|_{0, \Omega}^{2}\right\}^{1 / 2} .
\end{aligned}
$$

At this point, let us insist on the fact that the $h^{\alpha \beta}$ have to be in $L^{2}(\omega)$ which can be more restrictive than $\mathbf{h}$ being in the dual of $\mathbf{V}_{M}^{\#}(\omega)$. That is why, the results obtained by E. Sanchez-Palencia in [8] and [7] about this space are not enough to insure the convergence of $\overline{\mathbf{u}(\varepsilon)}$. To majorate the second integral of the rigthside of (10), we use again the Cauchy-Schwarz inequality, then the continuity of the trace on $\Gamma^{+}$and the majoration of (8), we conclude with inequality (5). Therefore,

$$
\left|\int_{\Gamma^{+}} h^{i+} u_{i}(\varepsilon) \varepsilon G d \Gamma\right| \leq \varepsilon\left\|\mathbf{h}^{+} G\right\|_{0, \Gamma^{+}}\|\mathbf{u}(\varepsilon)\|_{0, \Gamma^{+}} \leq c \varepsilon\|\mathbf{u}(\varepsilon)\|_{0, \Omega} \leq c \varepsilon\|\mathbf{u}(\varepsilon)\|_{1, \Omega} \leq c\left\{\sum_{i, j}\left\|e_{i \| j}(\varepsilon)\right\|_{0, \Omega}^{2}\right\}^{1 / 2} .
$$

Part (iii): (proof) The limits $e_{i \| j}$ found in part (ii) satisfy

$$
e_{1 \| 3}=0, e_{2 \| 3}=0 \text { and } e_{3 \| 3}=-\frac{\lambda}{\lambda+2 \mu} a^{\alpha \beta} e_{\alpha \| \beta} .
$$

In $\mathcal{P}(\varepsilon ; \Omega)$ we let $\mathbf{v}:=\varepsilon \mathbf{w}$, w being an arbitrary function in the space $\mathbf{V}(\Omega)$, and we let $\varepsilon$ approach zero; we obtain the equation

$$
\int_{\Omega}\left\{2 \mu a^{\alpha \sigma} e_{\sigma \| 3} \partial_{3} w_{\alpha}+\left(\lambda a^{\sigma \tau} e_{\sigma \| \tau}+(\lambda+2 \mu) e_{3 \| 3} \partial_{3} w_{3}\right)\right\} \sqrt{a} d x=0
$$

which, combined with (6), implies the result.

Part (iv): (no proof) The whole family $(\mathbf{u}(\varepsilon))_{\varepsilon>0}$ satisfies:

$$
\left\{\overline{e_{\alpha \| \beta}(\varepsilon)}-\gamma_{\alpha \beta}(\overline{\mathbf{u}(\varepsilon)})\right\} \longrightarrow 0 \text { in } L^{2}(\omega) \text { as } \varepsilon \rightarrow 0
$$

consequently, the subsequence considered in part (ii) satisfies:

$$
\gamma_{\alpha \beta}(\overline{\mathbf{u}(\varepsilon)}) \rightarrow \overline{e_{\alpha \| \beta}} \text { in } L^{2}(\omega) .
$$

Part $(v)$ : (partial proof) The limits $e_{\alpha \| \beta}$ found in part (ii) satisfy

$$
\int_{\omega} a^{\alpha \beta \sigma \tau} \overline{e_{\sigma \| \tau}} \gamma_{\alpha \beta}(\eta) \sqrt{a} d y=\int_{\omega} h^{\alpha \beta} \gamma_{\alpha \beta}(\eta) \sqrt{a} d y \text { for all } \eta \in \mathbf{V}(\omega),
$$

the functions $h^{\alpha \beta} \in L^{2}(\omega)$ being those used in the definition of admissible forces in Theorem 2. To prove the previous equation, we just need to check that

$$
\int_{\omega} h^{\alpha \beta} \gamma_{\alpha \beta}(\mathbf{v}) \sqrt{a} d y=\lim _{\varepsilon \rightarrow 0} \int_{\Gamma^{+}} h^{i+} v_{i} \sqrt{g(\varepsilon)} d \Gamma \text { for all } \mathbf{v} \in \mathbf{V}(\Omega) \text { independent of the transverse variable. }
$$

A function $\mathbf{v} \in \mathbf{V}(\Omega)$ independent of the transverse variable $x_{3}$ satisfies $\partial_{3} \mathbf{v}=\mathbf{0}$. That is why, using the same decomposition as in the proof of part $(i i)$, we have

$$
\int_{\Gamma^{+}} h^{i+} v_{i} \sqrt{g(\varepsilon)} d \Gamma=\int_{\omega} h^{\alpha \beta} \gamma_{\alpha \beta}(\mathbf{v}) \sqrt{a} d y+\varepsilon \int_{\Gamma^{+}} h^{i+} v_{i} G d \Gamma
$$


and the expected result when we let $\varepsilon \rightarrow 0$.

Part (vi): (no proof) The subsequence $(\mathbf{u}(\varepsilon))_{\varepsilon>0}$ found in part (ii) is such that

$$
\begin{aligned}
& \varepsilon \mathbf{u}(\varepsilon) \quad \rightarrow \mathbf{0} \text { in } \mathbf{H}^{1}(\Omega), \\
& \partial_{3} u_{\alpha}(\varepsilon) \quad \rightarrow 0 \text { in } L^{2}(\Omega),
\end{aligned}
$$

as $\varepsilon \rightarrow 0$. Furthermore, $e_{\alpha \| \beta}$ is independent of the transverse variable $x_{3}$.

Part (vii): (partial proof) The following strong convergences hold as $\varepsilon \rightarrow 0$ :

$$
\begin{array}{llll}
e_{i \| j}(\varepsilon) & \longrightarrow & e_{i \| j} & \text { in } L^{2}(\Omega), \\
\varepsilon \mathbf{u}(\varepsilon) & \longrightarrow & \mathbf{0} & \text { in } \mathbf{H}^{1}(\Omega), \\
\gamma_{\alpha \beta}(\overline{\mathbf{u}(\varepsilon)}) & \longrightarrow & \overline{e_{\alpha \| \beta}} & \text { in } L^{2}(\omega), \\
\overline{\mathbf{u}(\varepsilon)} & \longrightarrow & \zeta & \text { in } \mathbf{V}_{M}^{\#}(\omega) .
\end{array}
$$

To prove part (vii), we only need to prove the following result

$$
\lim _{\varepsilon \rightarrow 0} \int_{\Gamma^{+}} h^{i+} u_{i}(\varepsilon) \sqrt{g(\varepsilon)} d \Gamma=\int_{\omega} h^{\alpha \beta} \overline{e_{\alpha \| \beta}} \sqrt{a} d y .
$$

From the proof of part (ii), we know that

$$
\int_{\Gamma^{+}} h^{i+} u_{i}(\varepsilon) \sqrt{g(\varepsilon)} d \Gamma=\int_{\omega} h^{\alpha \beta} \gamma_{\alpha \beta}(\overline{\mathbf{u}(\varepsilon)}) \sqrt{a} d y+\frac{1}{2} \int_{\Omega}\left(1+x_{3}\right) \mathbf{h} \partial_{3} \mathbf{u}(\varepsilon) \sqrt{a} d x+\int_{\Gamma^{+}} h^{i+} \varepsilon u_{i}(\varepsilon) G d \Gamma
$$

and because of $(v i),(i v)$ and $(i i)$ we have:

$$
\lim _{\varepsilon \rightarrow 0} \frac{1}{2} \int_{\Omega}\left(1+x_{3}\right) \mathbf{h} \partial_{3} \mathbf{u}(\varepsilon) \sqrt{a} d x=0, \lim _{\varepsilon \rightarrow 0} \int_{\omega} h^{\alpha \beta} \gamma_{\alpha \beta}(\overline{\mathbf{u}(\varepsilon)}) \sqrt{a} d y=\int_{\omega} h^{\alpha \beta} \overline{e_{\alpha \| \beta}} \sqrt{a} d y \text { and } \lim _{\varepsilon \rightarrow 0} \int_{\Gamma^{+}} h^{i+} \varepsilon u_{i}(\varepsilon) G d \Gamma=0 .
$$

Hence, we just have to let $\varepsilon \rightarrow 0$ in (11) to get the announced result.

Part (viii): (no proof) The limit $\zeta \in \mathbf{V}_{M}^{\#}(\omega)$ found in part (ii) satisfies the equations

$$
B_{M}^{\#}(\zeta, \eta)=L_{M}^{\#}(\eta) \text { for all } \eta \in \mathbf{V}_{M}^{\#}(\omega),
$$

which have a unique solution. Consequently, the convergence

$$
\overline{\mathbf{u}(\varepsilon)} \longrightarrow \zeta \text { in } \mathbf{V}_{M}^{\#}(\omega)
$$

established in part (vii) holds for the whole family $(\overline{\mathbf{u}(\varepsilon)})_{\varepsilon>0}$.

Part (ix): (no proof) The following strong convergences hold:

$$
\begin{array}{llll}
\mathbf{u}(\varepsilon) & \longrightarrow & \mathbf{u} & \text { in } \mathbf{V}_{M}^{\#}(\Omega), \\
\partial_{3} u_{\alpha}(\varepsilon) & \longrightarrow & 0 & \text { in } L^{2}(\Omega)
\end{array}
$$

Part $(x)$ : (no proof) The whole family $(\mathbf{u}(\varepsilon))_{\varepsilon>0}$ converges strongly to $\mathbf{u}$ in the space $\mathbf{V}_{M}^{\#}(\Omega)$.

\section{Some applications}

In this section we first obtain a general system of PDE from the weak formulation (4), and afterwards, we carry through the study of this system in four representative cases. Since $\left(\gamma_{\alpha \beta}\right)$, the linearized change of metric tensor of $S$, depends on the geometry of the shell through the Christoffel symbols $\Gamma_{\alpha \beta}^{\sigma}$ and the curvature tensor $\left(b_{\alpha \beta}\right)$, see (1), the formulation 
of the PDE system depends on the geometry too. The choice of the coordinates set is very important to simplify the coupling between the unknowns. Indeed, if the geometry is hyperbolic, a parametrisation along the asymptotic lines leads to the following curvature tensor: $\left(b_{\alpha \beta}\right)=\left(\begin{array}{cc}0 & b_{12} \\ b_{12} & 0\end{array}\right)$, whereas if the geometry is parabolic, the tensor becomes $\left(b_{\alpha \beta}\right)=\left(\begin{array}{cc}b_{11} & 0 \\ 0 & 0\end{array}\right)$ when the first coordinate is along the asymptotic line (Sanchez-Hubert and Sanchez-Palencia [7]). Let us remind that an asymptotic line of a surface $S$ is a curve on $S$ having the property that at every point, the tangent vector is collinear with one of the asymptotic directions (directions for which the normal curvature is null).

Theorem 3 If the problem of unknowns $\left(h^{\alpha \beta}\right)$ (with $\left.h^{21}=h^{12}\right)$ :

$$
\left\{\begin{array}{l}
-\partial_{\beta}\left(h^{\alpha \beta} \sqrt{a}\right)-\Gamma_{\sigma \beta}^{\alpha}\left(h^{\sigma \beta} \sqrt{a}\right)=h^{\alpha} \sqrt{a} \quad \text { for } \alpha=1,2 \\
-b_{11} h^{11}-2 b_{12} h^{12}-b_{22} h^{22}=h^{3}
\end{array}\right.
$$

admits at least one solution such that

$$
\left\{\begin{array}{l}
h^{11} \in L^{2}(\omega), \partial_{1} h^{11} \in L^{2}(\omega), h^{11} n_{1}=0 \text { on } \partial \omega \backslash \gamma_{0}, \\
h^{22} \in L^{2}(\omega), \partial_{2} h^{22} \in L^{2}(\omega), h^{22} n_{2}=0 \text { on } \partial \omega \backslash \gamma_{0}, \\
h^{12} \in H^{1}(\omega), h^{12}=0 \text { on } \partial \omega \backslash \gamma_{0}
\end{array}\right.
$$

then $\mathbf{h}$ is admissible.

Proof. Let us assume that $h^{\alpha \beta}$ satisfy the regularity and boundary conditions (13). We integrate by part $\int_{\omega} h^{\alpha \beta} \gamma_{\alpha \beta}(\eta) \sqrt{a} d y$ with $\eta \in \mathbf{V}(\omega)$. The border integrals vanish because of the boundary conditions. By using the equations (12), we obtain $\int_{\omega} h^{\alpha \beta} \gamma_{\alpha \beta}(\eta) \sqrt{a} d y=\int_{\omega} h^{i} \eta_{i} \sqrt{a} d y$, so (4) is satisfied.

Before studying the existence of solutions for the PDE systems in four representative cases of partially or totally clamped, hyperbolic or parabolic shells, let us first remind two useful properties.

Property 1 Let $\omega:=] a, b[\times] c, d\left[\right.$ be an open bounded subset of $\mathbb{R}^{2}$ and $h$ be a function of $L^{2}(\omega)$. The function $f$ defined almost everywhere in $\omega$ by

$$
f(x, y):=\int_{a}^{x} h(t, y) d t
$$

is in $L^{2}(\omega)$ and satisfies

$$
\partial_{x} f=h \text { in } L^{2}(\omega), f=0 \text { on } x=a .
$$

A proof is given in Sanchez-Hubert and Sanchez-Palencia [7], p64.

Property 2 Let $\omega:=] a, b[\times] c, d\left[\right.$ be an open bounded subset of $\mathbb{R}^{2}$ and $h$ be a function of $L^{2}(\omega)$ such that $\partial_{y} h$ is in $L^{2}(\omega)$ too. Then, the function $f$ defined almost everywhere in $\omega$ by

$$
f(x, y):=\int_{a}^{x} h(t, y) d t
$$

is in $H^{1}(\omega)$ and satisfies

$$
\partial_{x} f=h \text { in } L^{2}(\omega), \partial_{y} f=\int_{a}^{x} \partial_{y} h(t, y) d t \text { in } L^{2}(\omega) \text { and } f=0 \text { on } x=a .
$$

Proof. Because of Property 1, we just need to prove that $\partial_{y} f=\int_{a}^{x} \partial_{y} h(t, y) d t$. To do so, we first prove the equality in the space of distibutions $\mathcal{D}^{\prime}(\omega)$, that is, we prove that for any $\varphi$ in $\mathcal{D}(\omega)$ :

$$
\int_{\omega} \partial_{y} f(x, y) \varphi(x, y) d x d y=\int_{\omega}\left(\int_{a}^{x} \partial_{y} h(t, y) d t\right) \varphi(x, y) d x d y
$$


Let $\varphi$ be in $\mathcal{D}(\omega), h$ and $\partial_{y} h$ be in $L^{2}(\omega)$ and $f(x, y):=\int_{a}^{x} h(t, y) d t$. By definition of the derivation in $\mathcal{D}^{\prime}(\omega)$ and by definition of $f$,

$$
\int_{\omega} \partial_{y} f(x, y) \varphi(x, y) d x d y=-\int_{\omega} f(x, y) \partial_{y} \varphi(x, y) d x d y=-\int_{\omega}\left(\int_{a}^{x} h(t, y) d t\right) \partial_{y} \varphi(x, y) d x d y
$$

From the Ostrogradsky formula $\int_{\omega} \partial_{x} u v d x d y=-\int_{\omega} u \partial_{x} v d x d y+\int_{\partial \omega} u v \nu_{x} d l$, that can be used as soon as $\partial_{x} u$ and $\partial_{x} v$ are in $L^{2}(\omega)$, we deduce that

$$
-\int_{\omega}\left(\int_{a}^{x} h(t, y) d t\right) \partial_{y} \varphi(x, y) d x d y=\int_{\omega}\left(\int_{b}^{x} \partial_{y} \varphi(t, y) d t\right) h(x, y) d x d y-\int_{\partial \omega}\left(\int_{a}^{x} h(t, y) d t\right)\left(\int_{b}^{x} \partial_{y} \varphi(t, y) d t\right) \nu_{x} d l
$$

But, on the borders $y=c$ and $y=d, \nu_{x}=0$, on the border $x=a, \int_{a}^{x} h(t, y) d t=0$ and on the border $x=b$, $\int_{b}^{x} \partial_{y} \varphi(t, y) d t=0$, so that the border integral vanishes. Because of $\varphi$ 's regularity, we can permutate $\int$ and $\partial_{y}$ and use once more an Ostrogradsky formula so that

$$
\int_{\omega} \partial_{y}\left(\int_{b}^{x} \varphi(t, y) d t\right) h(x, y) d x d y=-\int_{\omega}\left(\int_{b}^{x} \varphi(t, y) d t\right) \partial_{y} h(x, y) d x d y+\int_{\partial \omega} h(x, y)\left(\int_{b}^{x} \varphi(t, y) d t\right) \nu_{y} d l .
$$

On the borders $x=a$ and $x=b, \nu_{y}=0$, on the borders $y=c$ and $y=d, \varphi=0$, so that the border integral vanishes again. We integrate by parts again and obtain

$$
-\int_{\omega}\left(\int_{b}^{x} \varphi(t, y) d t\right) \partial_{y} h(x, y) d x d y=\int_{\omega}\left(\int_{a}^{x} \partial_{y} h(t, y) d t\right) \varphi(t, y) d x d y-\int_{\partial \omega}\left(\int_{a}^{x} \partial_{y} h(t, y) d t\right)\left(\int_{b}^{x} \varphi(t, y) d t\right) \nu_{x} d l .
$$

Since the border integral is again equal to 0 , we have the expected equality in $\mathcal{D}^{\prime}(\omega)$. To conclude, from Property $\mathbf{1}$, we know that $\int_{a}^{x} \partial_{y} h(t, y) d t$ is in $L^{2}(\omega)$, so the equality takes place in $L^{2}(\omega)$.

\subsection{Hyperbolic shell totally clamped}

Let us suppose that the middle surface of the shell is the following portion of a hyperbolic paraboloid

$$
\mathcal{H P}:=\left\{\left(x_{1}, x_{2}, x_{3}\right) \in \mathbb{R}^{3}, \frac{x_{3}}{a_{3}}=\frac{x_{1}^{2}}{a_{1}^{2}}-\frac{x_{2}^{2}}{a_{2}^{2}} \text { with }-x_{0}<x_{1}<x_{0} \text { and }-y_{0}<x_{2}<y_{0}\right\}
$$

and that the shell is totally clamped. We choose a parametrisation of $\mathcal{H P}$ along the asymptotic lines, so that the mapping $\theta$ is

$$
\begin{aligned}
& \theta: \quad \bar{\omega} \quad \longrightarrow \mathbb{R}^{3} \\
& (\varphi, \psi) \longmapsto\left(\frac{a_{1}}{2}(\varphi+\psi), \frac{a_{2}}{2}(\varphi-\psi), a_{3} \varphi \psi\right)
\end{aligned}
$$

where $\omega$ is the subset

$$
\omega:=\left\{(\varphi, \psi) \in \mathbb{R}^{2}, \varphi-\frac{2 y_{0}}{a_{2}}<\psi<\varphi+\frac{2 y_{0}}{a_{2}} \text { and }-\varphi-\frac{2 x_{0}}{a_{1}}<\psi<-\varphi+\frac{2 x_{0}}{a_{1}}\right\} .
$$

The border is then represented by

$$
\begin{aligned}
& \partial \omega:=\left\{\left(\varphi,+\varphi-\frac{2 y_{0}}{a_{2}}\right), \varphi \in\right]-\frac{x_{0}}{a_{1}}+\frac{y_{0}}{a_{2}},+\frac{x_{0}}{a_{1}}+\frac{y_{0}}{a_{2}}[\} \cup \\
& \left.\left.\left\{\left(\varphi,+\varphi+\frac{2 y_{0}}{a_{2}}\right), \varphi \in\right]-\frac{x_{0}}{a_{1}}-\frac{y_{0}}{a_{2}},+\frac{x_{0}}{a_{1}}-\frac{y_{0}}{a_{2}}\right]\right\} \cup \\
& \left.\left\{\left(\varphi,-\varphi-\frac{2 x_{0}}{a_{1}}\right), \varphi \in-\frac{x_{0}}{a_{1}}-\frac{y_{0}}{a_{2}},-\frac{x_{0}}{a_{1}}+\frac{y_{0}}{a_{2}}\right\}\right\} \cup \\
& \left\{\left(\varphi,-\varphi+\frac{2 x_{0}}{a_{1}}\right), \varphi \in\right]+\frac{x_{0}}{a_{1}}-\frac{y_{0}}{a_{2}},+\frac{x_{0}}{a_{1}}+\frac{y_{0}}{a_{2}}[\} \text {. }
\end{aligned}
$$

In these coordinates, the second fundamental form $\left(b_{\alpha \beta}\right)_{\alpha \beta}$ verifies,

$$
b_{11}=b_{22}=0 \text { and } b_{12} \neq 0 \text {, }
$$



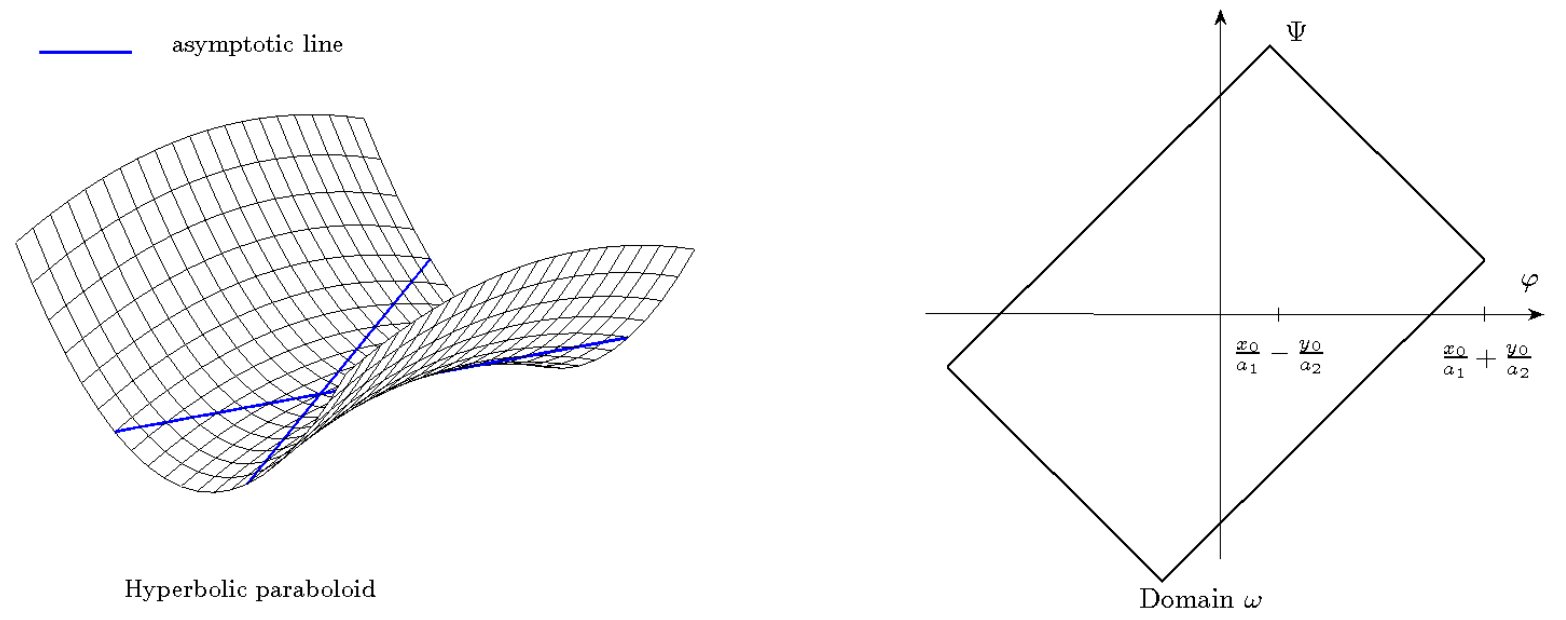

Figure 1: Hyperbolic shell totally clamped

the Christoffel symbols are such that,

$$
\Gamma_{11}^{1}=\Gamma_{22}^{2}=\Gamma_{22}^{1}=\Gamma_{11}^{2}=0, \Gamma_{12}^{1} \neq 0 \text { and } \Gamma_{12}^{2} \neq 0
$$

and the Jacobian $\sqrt{a}$ is different from 0 . The displacement field $\eta=\left(\eta_{i}\right)$ is looked for in $\mathbf{H}_{0}^{1}(\omega)$. Under these assumptions, the admissibility condition given by (4) becomes : find $h^{\alpha \beta}$ in $L^{2}(\omega)$, such that for all $\eta=\left(\eta_{i}\right) \in \mathbf{H}_{0}^{1}(\omega)$,

$$
\int_{\omega} h^{i} \eta_{i} \sqrt{a} d y=\int_{\omega}\left(h^{11} \partial_{\varphi} \eta_{1}+h^{22} \partial_{\psi} \eta_{2}+h^{12}\left(\partial_{\psi} \eta_{1}+\partial_{\varphi} \eta_{2}-2 \Gamma_{12}^{1} \eta_{1}-2 \Gamma_{12}^{2} \eta_{2}-2 b_{12} \eta_{3}\right)\right) \sqrt{a} d y
$$

Theorem 4 The surface force $\mathbf{h}=\left(h^{i}\right) \in \mathbf{L}^{2}(\omega)$ is admissible if $h^{3}$ is in $H^{1}(\omega)$.

Proof. Let $h^{1}, h^{2}$ be in $L^{2}(\omega), h^{3}$ be in $H^{1}(\omega)$. Let

$$
\begin{aligned}
& V_{\varphi}(\omega):=\left\{h \in L^{2}(\omega), \partial_{\varphi} h \in L^{2}(\omega)\right\}, \\
& V_{\psi}(\omega):=\left\{h \in L^{2}(\omega), \partial_{\psi} h \in L^{2}(\omega)\right\} .
\end{aligned}
$$

From Theorem 3, we know that $\mathbf{h}$ is admissible if there exist $h^{11}$ in $V_{\varphi}(\omega), h^{22}$ in $V_{\psi}(\omega)$ and $h^{12}$ in $H^{1}(\omega)$ such that :

$$
\left\{\begin{array}{l}
-\partial_{\beta}\left(h^{\alpha \beta} \sqrt{a}\right)-\Gamma_{12}^{\alpha}\left(h^{12} \sqrt{a}\right)=h^{\alpha} \sqrt{a} \quad \text { for } \alpha=1,2 \\
-2 b_{12} h^{12}=h^{3}
\end{array}\right.
$$

$h^{12}=-\frac{h^{3}}{2 b_{12}}$ is appropriate since it is in $H^{1}(\omega)$. We substitute this function for $h^{12}$ and thus obtain two uncoupled PDE

$$
\partial_{\varphi}\left(h^{11} \sqrt{a}\right)=\hat{f}_{1} \text { in } L^{2}(\omega)
$$

where

$$
\hat{f}_{1}:=-h^{1} \sqrt{a}+\partial_{\psi}\left(\frac{1}{2 b_{12}} h^{3} \sqrt{a}\right)+\frac{\Gamma_{12}^{1}}{b_{12}} h^{3} \sqrt{a} \in L^{2}(\omega),
$$


and,

$$
\partial_{\psi}\left(h^{22} \sqrt{a}\right)=\hat{f}_{2} \text { in } L^{2}(\omega)
$$

where

$$
\hat{f}_{2}:=-h^{2} \sqrt{a}+\partial_{\varphi}\left(\frac{1}{2 b_{12}} h^{3} \sqrt{a}\right)+\frac{\Gamma_{12}^{2}}{b_{12}} h^{3} \sqrt{a} \in L^{2}(\omega) .
$$

For almost all $(\varphi, \psi) \in \omega$, let

$$
g(\varphi, \psi):=\frac{1}{\sqrt{a}} \int_{0}^{\varphi} \hat{f}_{1}(\eta, \psi) d \eta
$$

then of course $\partial_{\varphi}(g \sqrt{a})=\hat{f}_{1}$ in $L^{2}(\omega)$. So, we just need to prove that $g$ is in $L^{2}(\omega)$ to have $g$ in $V_{\varphi}(\omega)$ and thereby get the result by letting $h^{11}=g$. From the Cauchy-Schwarz inequality, for almost all $(\varphi, \psi) \in \omega$, we have :

$$
\int_{0}^{\varphi} \hat{f}_{1}(\eta, \psi) d \eta \leq \sqrt{\left|\int_{0}^{\varphi} \hat{f}_{1}^{2}(\eta, \psi) d \eta\right|} \sqrt{\left|\int_{0}^{\varphi} 1^{2} d \eta\right|}
$$

so that

$$
\left(\int_{0}^{\varphi} \hat{f}_{1}(\eta, \psi) d \eta\right)^{2} \leq|\varphi|\left|\int_{0}^{\varphi} \hat{f}_{1}^{2}(\eta, \psi) d \eta\right|
$$

Let $\tilde{\omega}$ be the following open subset which contains $\omega$

$$
\tilde{\omega}:=]-\frac{x_{0}}{a_{1}}-\frac{y_{0}}{a_{2}}, \frac{x_{0}}{a_{1}}+\frac{y_{0}}{a_{2}}[\times]-\frac{x_{0}}{a_{1}}-\frac{y_{0}}{a_{2}}, \frac{x_{0}}{a_{1}}+\frac{y_{0}}{a_{2}}[.
$$

and let $\tilde{f}_{1}$ be the extension by zeros of $\hat{f}_{1}$ to $\tilde{\omega}$. Since $\hat{f}_{1}$ is in $L^{2}(\omega)$ then $\tilde{f}_{1}$ is in $L^{2}(\tilde{\omega})$ too and obviously, if the integrals exist, they verify

$$
\int_{\omega}\left(\int_{0}^{\varphi} \hat{f}_{1}(\eta, \psi) \partial \eta\right)^{2} d y \leq \int_{\tilde{\omega}}\left(\int_{0}^{\varphi} \hat{f}_{1}(\eta, \psi) \partial \eta\right)^{2} d y
$$

For all $(\varphi, \psi) \in \tilde{\omega},|\varphi| \leq \frac{x_{0}}{a_{1}}+\frac{y_{0}}{a_{2}}$, so, after integrating (15) on $\tilde{\omega}$, we obtain the following bounding :

$$
\int_{\tilde{\omega}}\left(\int_{0}^{\varphi} \hat{f}_{1}(\eta, \psi) \partial \eta\right)^{2} d y \leq\left(\frac{x_{0}}{a_{1}}+\frac{y_{0}}{a_{2}}\right) \int_{\tilde{\omega}}\left(\int_{-\frac{x_{0}}{a_{1}}-\frac{y_{0}}{a_{2}}}^{\frac{x_{0}}{a_{1}}+\frac{y_{0}}{a_{2}}} \tilde{f}_{1}^{2}(\eta, \psi) d \eta\right) d y .
$$

From the Tonelli Theorem we have :

$$
\int_{\tilde{\omega}}\left(\int_{-\frac{x_{0}}{a_{1}}-\frac{y_{0}}{a_{2}}}^{\frac{x_{0}}{a_{1}}+\frac{y_{0}}{a_{2}}} \tilde{f}_{1}^{2}(\eta, \psi) d \eta\right) d y=\int_{-\frac{x_{0}}{a_{1}}-\frac{y_{0}}{a_{2}}}^{\frac{x_{0}}{a_{1}}+\frac{y_{0}}{a_{2}}}\left(\int_{\tilde{\omega}} \tilde{f}_{1}^{2}(\eta, \psi) d y\right) d \eta=\left(\frac{x_{0}}{a_{1}}+\frac{y_{0}}{a_{2}}\right)\left\|\tilde{f}_{1}\right\|_{0, \tilde{\omega}}^{2},
$$

So, since $\tilde{f}_{1}$ is in $L^{2}(\tilde{\omega})$, we have the expected bounding

$$
\int_{\omega}\left(\int_{0}^{\varphi} \hat{f}_{1}(\eta, \psi) \partial \eta\right)^{2} d y<\infty
$$

which insures that $g$ is in $L^{2}(\omega)$, and, consequently, in $V_{\varphi}(\omega)$. We proceed the same way to build $h^{22}$ in $V_{\psi}(\omega)$.

\subsection{Hyperbolic shell partially clamped}

Let us suppose that the middle surface of the shell is a portion of hyperbolod $\mathcal{H}$ and that it is clamped along its entire "lower" face $\Gamma_{0}$. Let the cartesian equations of $\mathcal{H}$ be

$$
\frac{x_{1}^{2}}{a_{1}^{2}}+\frac{x_{2}^{2}}{a_{2}^{2}}-\frac{x_{3}^{2}}{a_{3}^{2}}=1 \text { and } z_{0} \leq x_{3} \leq z_{1} .
$$



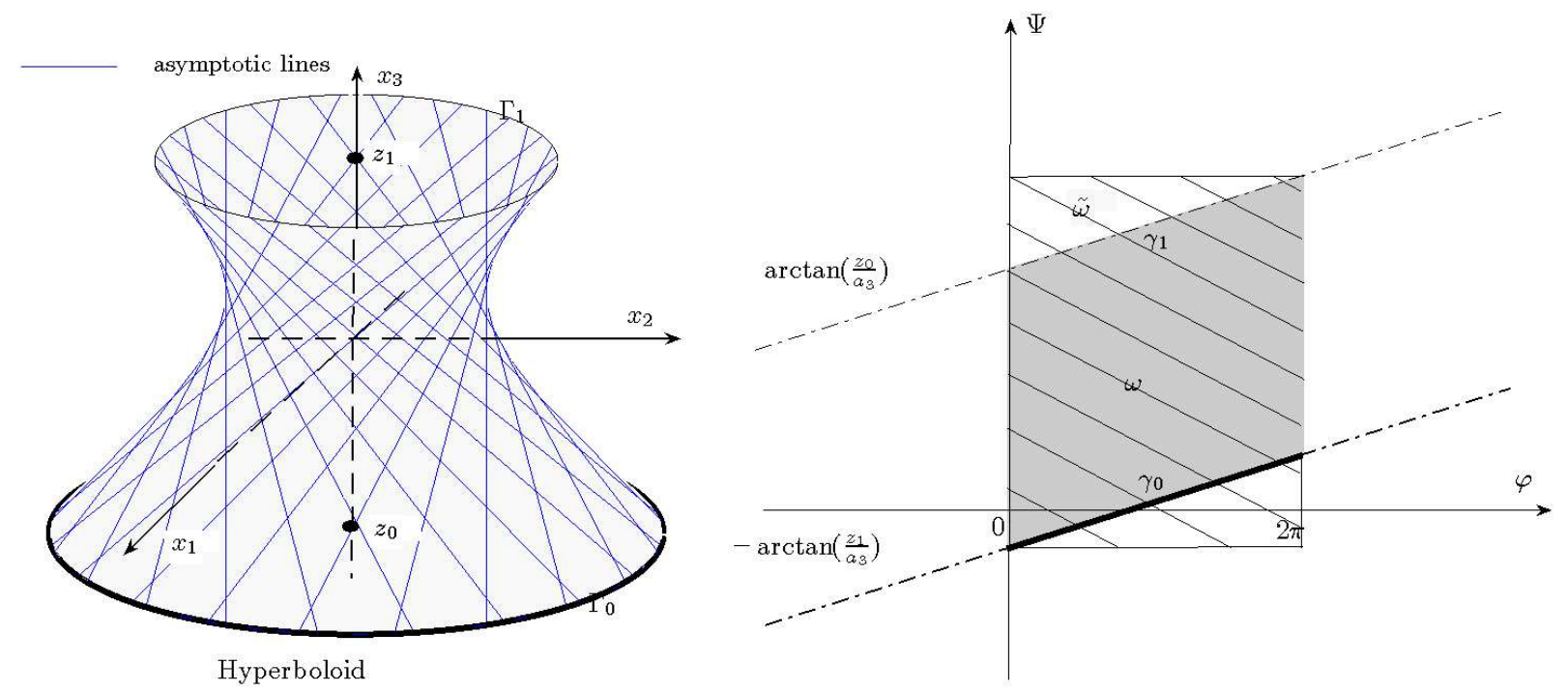

Figure 2: Hyperbolic shell partially clamped

We choose a parametrisation of $\mathcal{H}$ along the asymptotic lines, so that

$$
\begin{aligned}
\theta: \bar{\omega} & \longrightarrow \mathbb{R}^{3} \\
(\varphi, \psi) & \longmapsto\left(a_{1} \frac{\cos (\varphi+\psi)}{\cos (\varphi-\psi)}, a_{2} \frac{\sin (\varphi+\psi)}{\cos (\varphi-\psi)}, a_{3} \tan (\varphi-\psi)\right)
\end{aligned}
$$

where

$$
\begin{aligned}
& \omega:=\{(\varphi, \psi), \varphi \in] 0, \pi[, \psi \in] \varphi-\arctan \frac{z_{1}}{a_{3}}, \varphi-\arctan \frac{z_{0}}{a_{3}}[\} \\
& :=\{(\varphi, \psi), \psi \in]-\arctan \frac{z_{1}}{a_{3}}, \pi-\arctan \frac{z_{0}}{a_{3}},[, \varphi \in] \max \left(\psi+\arctan \frac{z_{0}}{a_{3}}, 0\right), \min \left(\psi+\arctan \frac{z_{1}}{a_{3}}, \pi\right)[\},
\end{aligned}
$$

the upper border $\Gamma_{1}$ is represented by

$$
\gamma_{1}:=\left\{\left(\varphi, \varphi-\arctan \frac{z_{1}}{a_{3}}\right), \varphi \in\right] 0, \pi[\}:=\left\{\left(\psi+\arctan \frac{z_{1}}{a_{3}}, \psi\right), \psi \in\right]-\arctan \frac{z_{1}}{a_{3}}, \pi-\arctan \frac{z_{0}}{a_{3}}[\},
$$

and where the lower border $\Gamma_{0}$ is represented by

$$
\gamma_{0}:=\left\{\left(\varphi, \varphi-\arctan \frac{z_{0}}{a_{3}}\right), \varphi \in\right] 0, \pi[\}:=\left\{\left(\psi+\arctan \frac{z_{0}}{a_{3}}, \psi\right), \psi \in\right]-\arctan \frac{z_{1}}{a_{3}}, \pi-\arctan \frac{z_{0}}{a_{3}}[\} .
$$

Let $\tilde{\omega}$ be the open subset

$$
\tilde{\omega}:=] \arctan \frac{z_{0}}{a_{3}}-\arctan \frac{z_{1}}{a_{3}}, \pi[\times] \arctan \frac{z_{0}}{a_{3}}-\arctan \frac{z_{1}}{a_{3}}, \pi-\arctan \frac{z_{0}}{a_{3}}[.
$$

The displacement field is looked for in $\mathbf{V}(\omega)=\left\{\mathbf{v} \in \mathbf{H}^{1}(\omega), \mathbf{v} \pi\right.$-periodic, $\mathbf{v}=\mathbf{0}$ on $\left.\gamma_{0}\right\}$. The second fundamental form $\left(b_{\alpha \beta}\right)_{\alpha \beta}$ is $\pi$-periodic, such that,

$$
b_{11}=b_{22}=0 \text { and } b_{12} \neq 0 \text {, }
$$


the Christoffel symbols are $\pi$-periodic, such that,

$$
\Gamma_{11}^{1}=-\Gamma_{22}^{2}=2 \tan (\varphi-\psi), \Gamma_{22}^{1}=\Gamma_{11}^{2}=0, \Gamma_{12}^{1} \neq 0 \text { and } \Gamma_{12}^{2} \neq 0
$$

and the jacobian $\sqrt{a}$ is $\pi$-periodic, different from 0 . Under these assumptions, the admissibilty condition (4) becomes $h^{\alpha \beta} \in L^{2}(\omega)$, such that :

$$
\begin{aligned}
\int_{\omega} h^{i} \eta_{i} \sqrt{a} d y= & \int_{\omega}\left(h^{11}\left(\partial_{\varphi} \eta_{1}-2 \tan (\varphi-\psi) \eta_{1}\right)+h^{22}\left(\partial_{\psi} \eta_{2}+2 \tan (\varphi-\psi) \eta_{2}\right)\right) \sqrt{a} d y \\
& +\int_{\omega} h^{12}\left(\partial_{\psi} \eta_{1}+\partial_{\varphi} \eta_{2}-2 \Gamma_{12}^{1} \eta_{1}-2 \Gamma_{12}^{2} \eta_{2}-2 b_{12} \eta_{3}\right) \sqrt{a} d y \text { for all } \eta \in \mathbf{V}(\omega) .
\end{aligned}
$$

Theorem $\mathbf{5}$ The $\pi$-periodic surface force $\mathbf{h}=\left(h^{i}\right) \in \mathbf{L}^{2}(\omega)$ is admissible if

$$
\left\{\begin{array}{l}
h^{3} \in H^{1}(\omega), \partial_{\varphi \psi} h^{3} \in L^{2}(\omega), h^{3}=0 \text { on } \gamma_{1} \\
\partial_{\varphi} h^{1} \in L^{2}(\omega) \\
\partial_{\psi} h^{2} \in L^{2}(\omega)
\end{array}\right.
$$

Proof. Let $\mathbf{h}=\left(h^{i}\right)$ be a $\pi$-periodic function of $\mathbf{L}^{2}(\omega)$ satisfying (17) and let

$$
\begin{aligned}
& V_{\varphi}(\omega):=\left\{h \in L^{2}(\omega), \partial_{\varphi} h \in L^{2}(\omega), h \pi \text {-periodic and } h \nu_{\varphi}=0 \text { on } \gamma_{1}\right\}, \\
& V_{\psi}(\omega):=\left\{h \in L^{2}(\omega), \partial_{\psi} h \in L^{2}(\omega), h \pi \text {-periodic and } h \nu_{\psi}=0 \text { on } \gamma_{1}\right\} .
\end{aligned}
$$

From Theorem 3 we know that $\mathbf{h}$ is admissible if there exist $h^{11}$ in $V_{\varphi}(\omega), h^{22}$ in $V_{\psi}(\omega)$ and $h^{12}$ in $V_{\varphi}(\omega) \cap V_{\psi}(\omega)$ such that :

$$
\left\{\begin{array}{l}
-\partial_{\varphi}\left(h^{11} \sqrt{a}\right)-\partial_{\psi}\left(h^{12} \sqrt{a}\right)-\Gamma_{11}^{1} h^{11} \sqrt{a}-2 \Gamma_{12}^{1} h^{12} \sqrt{a}=h^{1} \sqrt{a} \\
-\partial_{\varphi}\left(h^{12} \sqrt{a}\right)-\partial_{\psi}\left(h^{22} \sqrt{a}\right)-\Gamma_{22}^{2} h^{22} \sqrt{a}-2 \Gamma_{12}^{2} h^{12} \sqrt{a}=h^{2} \sqrt{a} \\
-2 b_{12} h^{12}=h^{3}
\end{array}\right.
$$

$h^{12}=-\frac{h^{3}}{2 b_{12}}$ is appropriate since it is in $V_{\varphi}(\omega) \cap V_{\psi}(\omega)$. We substitute $h^{12}$ and thus obtain two uncoupled PDE :

$$
-\partial_{\varphi}\left(h^{11} \sqrt{a}\right)-2 \tan (\varphi-\psi)\left(h^{11} \sqrt{a}\right)=\left(h^{1} \sqrt{a}\right)-\partial_{\psi}\left(\frac{1}{2 b_{12}} h^{3} \sqrt{a}\right)-\frac{\Gamma_{12}^{1}}{b_{12}} h^{3} \sqrt{a}
$$

and

$$
-\partial_{\psi}\left(h^{22} \sqrt{a}\right)+2 \tan (\varphi-\psi)\left(h^{22} \sqrt{a}\right)=\left(h^{2} \sqrt{a}\right)-\partial_{\psi}\left(\frac{1}{2 b_{12}} h^{3} \sqrt{a}\right)-\frac{\Gamma_{12}^{2}}{b_{12}} h^{3} \sqrt{a} .
$$

Let us notice that

$$
\partial_{\varphi}\left(h^{11} \sqrt{a}\right)+2 \tan (\varphi-\psi)\left(h^{11} \sqrt{a}\right)=\cos ^{2}(\varphi-\psi) \partial_{\varphi}\left(\frac{h^{11} \sqrt{a}}{\cos ^{2}(\varphi-\psi)}\right)
$$

and that

$$
\partial_{\psi}\left(h^{22} \sqrt{a}\right)-2 \tan (\varphi-\psi)\left(h^{22} \sqrt{a}\right)=\cos ^{2}(\varphi-\psi) \partial_{\psi}\left(\frac{h^{22} \sqrt{a}}{\cos ^{2}(\varphi-\psi)}\right)
$$

so if we let

$$
\hat{f}_{1}:=\frac{1}{\cos ^{2}(\varphi-\psi)}\left(-\left(h^{1} \sqrt{a}\right)+\partial_{\psi}\left(\frac{1}{2 b_{12}}\left(h^{3} \sqrt{a}\right)\right)+\frac{\Gamma_{12}^{1}}{b_{12}}\left(h^{3} \sqrt{a}\right)\right)
$$

and

$$
\hat{f}_{2}:=\frac{1}{\cos ^{2}(\varphi-\psi)}\left(-\left(h^{2} \sqrt{a}\right)+\partial_{\varphi}\left(\frac{1}{2 b_{12}}\left(h^{3} \sqrt{a}\right)\right)+\frac{\Gamma_{12}^{2}}{b_{12}}\left(h^{3} \sqrt{a}\right)\right) .
$$

$\hat{f}_{1}$ and $\hat{f}_{2}$ are both $\pi$-periodic and, because of (17), both in $L^{2}(\omega)$. Therefore, $\mathbf{h}$ is admissible if we can find $h^{11} \in V_{\varphi}(\omega)$ such that

$$
\partial_{\varphi}\left(\frac{h^{11} \sqrt{a}}{\cos ^{2}(\varphi-\psi)}\right)=\hat{f}_{1} \text { in } L^{2}(\omega)
$$


and $h^{22} \in V_{\psi}(\omega)$ such that

$$
\partial_{\psi}\left(\frac{h^{22} \sqrt{a}}{\cos ^{2}(\varphi-\psi)}\right)=\hat{f}_{2} \text { in } L^{2}(\omega)
$$

Let $\tilde{f}_{1}$ be the extension by zeros of $\hat{f}_{1}$ to $\tilde{\omega}$. Since $\hat{f}_{1}$ is in $L^{2}(\omega)$ then $\tilde{f}_{1}$ is in $L^{2}(\tilde{\omega})$. For almost all $(\varphi, \psi) \in \omega$, let

$$
g(\varphi, \psi):=\frac{\cos ^{2}(\varphi-\psi)}{\sqrt{a}} \int_{\psi+\arctan \frac{z_{0}}{a_{3}}}^{\varphi} \hat{f}_{1}(\eta, \psi) d \eta,
$$

and let us prove that $g$ is in $V_{\varphi}(\omega)$. In order to do that, we just have to prove that the integral $\int_{\psi+\arctan \frac{z_{0}}{a_{3}}}^{\varphi} \hat{f}_{1}(\eta, \psi) d \eta$ is in $L^{2}(\omega)$ and vanishes on $\gamma_{1}$. The second point is obvious. From the Cauchy-Schwarz inequality, for almost all $(\varphi, \psi) \in \omega$, we have :

$$
\left(\int_{\psi+\arctan \frac{z_{0}}{a_{3}}}^{\varphi} \hat{f}_{1}(\eta, \psi) d \eta\right)^{2} \leq\left|\int_{\psi+\arctan \frac{z_{0}}{a_{3}}}^{\varphi} \hat{f}_{1}^{2}(\eta, \psi) d \eta \int_{\psi+\arctan \frac{z_{0}}{a_{3}}}^{\varphi} 1^{2} d \eta\right| .
$$

Since for all $(\varphi, \psi) \in \omega,\left|\varphi-\psi-\arctan \frac{z_{0}}{a_{3}}\right| \leq \pi$ and since $\hat{f}_{1}$ is $\pi$-periodic then,

$$
\left(\int_{\psi+\arctan \frac{z_{0}}{a_{3}}}^{\varphi} \hat{f}_{1}(\eta, \psi) d \eta\right)^{2} \leq \pi \int_{0}^{\pi} \hat{f}_{1}^{2}(\eta, \psi) d \eta
$$

moreover, as $\omega \subset \tilde{\omega}$, we have the following bounding :

$$
\int_{\omega}\left(\int_{\psi+\arctan \frac{z_{0}}{a_{3}}}^{\varphi} \hat{f}_{1}(\eta, \psi) \partial \eta\right)^{2} d y \leq \pi \int_{\tilde{\omega}}\left(\int_{0}^{\pi} \tilde{f}_{1}^{2}(\eta, \psi) d \eta\right) d y .
$$

We conclude with the Tonelli Theorem that :

$$
\pi \int_{\tilde{\omega}}\left(\int_{0}^{\pi} \tilde{f}_{1}^{2}(\eta, \psi) d \eta\right) d y=\pi^{2}\left\|\tilde{f}_{1}\right\|_{0, \tilde{\omega}}^{2}<\infty
$$

so, $h^{11}=g$ is a suitable solulution. We proceed the same way to find $h^{22}$ in $V_{\psi}(\omega)$.

\subsection{Parabolic shell totally clamped}

Let us suppose the shell $\mathcal{C}$ is a portion of a cone which is subjected to a boundary condition of place along its whole latteral face.

$$
\mathcal{C}:=\left\{\left(x_{1}, x_{2}, x_{3}\right) \in \mathbb{R}^{3}, x_{1}=r \cos \theta, x_{2}=r \sin \theta, x_{3}=r \cot \varphi,(r, \theta) \in \bar{\omega}\right\}
$$

where

$$
\omega:=] r_{0}, r_{1}[\times] \theta_{0}, \theta_{1}[\text { and } \varphi \in] 0, \pi / 2[\text {. }
$$

The admissibilty condition (4) becomes : find $h^{\alpha \beta}$ in $L^{2}(\omega)$, such that for all $\eta=\left(\eta_{i}\right) \in \mathbf{H}_{0}^{1}(\omega)$,

$$
\int_{\omega} h^{i} \eta_{i} \sqrt{a} d y=\int_{\omega}\left(h^{11} \partial_{r} \eta_{1}+h^{22}\left(\partial_{\theta} \eta_{2}+r \sin ^{2} \varphi \eta_{2}-r \cos \varphi \eta_{3}\right)+h^{12}\left(\partial_{\theta} \eta_{1}+\partial_{r} \eta_{2}-\frac{2}{r} \eta_{2}\right)\right) \sqrt{a} d y
$$

where $\sqrt{a}=\frac{r}{\sin \varphi}$.

Theorem 6 The surface force $\mathbf{h}=\left(h^{i}\right) \in \mathbf{L}^{2}(\omega)$ is admissible as soon as

$$
\partial_{\theta} h^{2}, \partial_{\theta} h^{3} \text { and } \partial_{\theta \theta} h^{3} \text { are in } L^{2}(\omega) .
$$



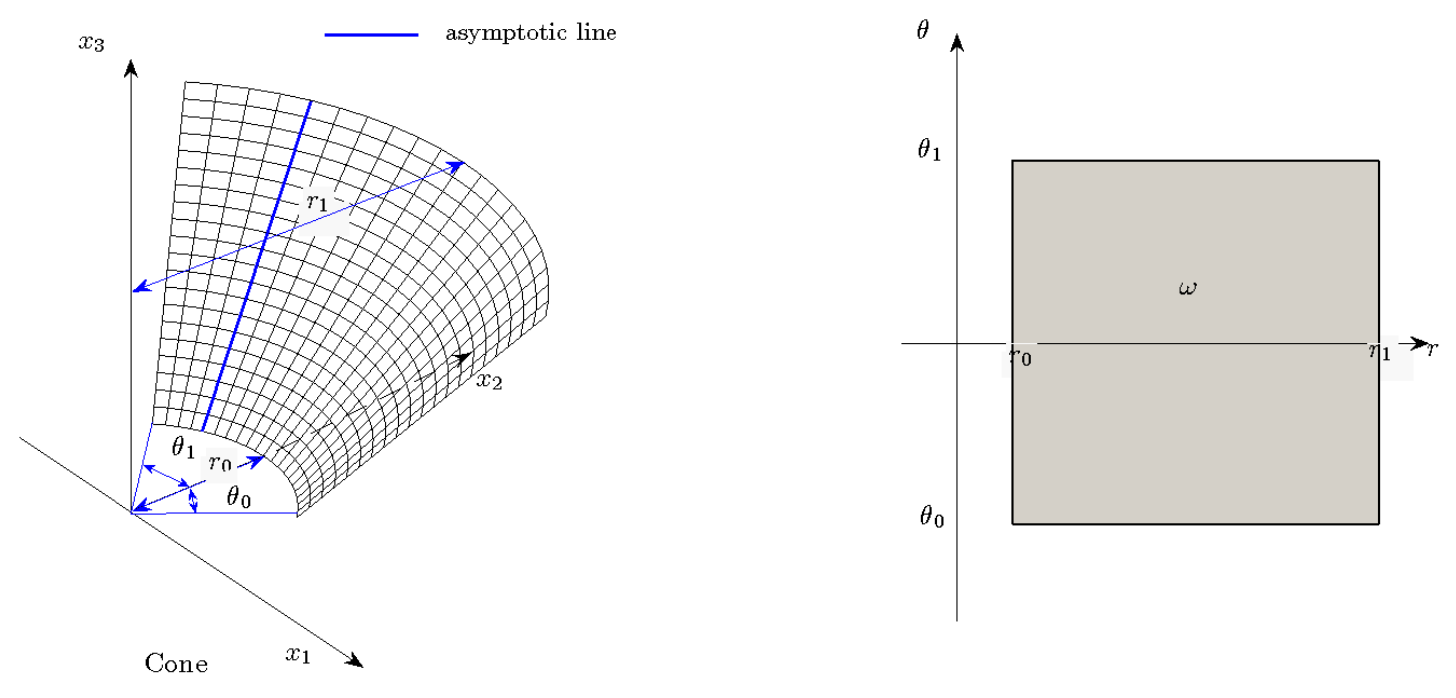

Figure 3: Parabolic shell totally clamped

Proof. Let $\mathbf{h}=\left(h^{i}\right)$ be a function of $\mathbf{L}^{2}(\omega)$ satisfying (19). If we can find $h^{11}, h^{22}$ in $L^{2}(\omega)$ such that $\partial_{r} h^{11}$ and $\partial_{\theta} h^{22}$ are in $L^{2}(\omega)$, and $h^{12}$ in $H^{1}(\omega)$ satisfying

$$
\left\{\begin{array}{l}
-\partial_{r}\left(h^{11} \sqrt{a}\right)-\partial_{\theta}\left(h^{12} \sqrt{a}\right)+r \sin ^{2} \varphi h^{12} \sqrt{a}=h^{1} \sqrt{a} \\
-\partial_{r}\left(h^{12} \sqrt{a}\right)-\partial_{\theta}\left(h^{22} \sqrt{a}\right)-\frac{2}{r} h^{12} \sqrt{a}=h^{2} \sqrt{a} \\
-r \cos \varphi h^{22}=h^{3}
\end{array}\right.
$$

then $\mathbf{h}$ is admissible. Let $h^{22}=-\frac{h^{3}}{r \cos \varphi}$, both $h^{22}$ and $\partial_{\theta} h^{22}$ are in $L^{2}(\omega)$. Let us point out that $\partial_{r} \eta-\frac{2}{r} \eta=r^{2} \partial_{r}\left(\frac{\eta}{r^{2}}\right)$ and substitute $h^{22}$ in (20), then, $h^{12}$ satisfies

$$
-\partial_{r}\left(r^{3} h^{12}\right)=h^{2} r^{3}-\frac{r^{2}}{\cos \varphi} \partial_{\theta} h^{3} \text { in } L^{2}(\omega)
$$

A possibility for $h^{12}$ is

$$
h^{12}=\frac{1}{r^{3}} \int_{r_{0}}^{r}\left(-h^{2} r^{3}+\frac{r^{2}}{\cos \varphi} \partial_{\theta} h^{3}\right) d r .
$$

With this choice, $h^{12}, \partial_{r} h^{12}$ and $\partial_{\theta} h^{12}$ are in $L^{2}(\omega)$ as shown by Property 1 and Property 2. Finally, replacing $h^{12}$ and $h^{22}$ in (20) and integrating by parts, we notice that if there exists $h^{11} \in L^{2}(\omega)$ such that

$$
-\partial_{r}\left(r h^{11}\right)=r h^{1}+\frac{1}{r^{2}} \int_{r_{0}}^{r} \partial_{\theta}\left(-h^{2} r^{3}+\frac{r^{2}}{\cos \varphi} \partial_{\theta} h^{3}\right) d r+r \frac{\sin ^{2} \varphi}{\cos \varphi} h^{3} \text { in } L^{2}(\omega),
$$

the admissibility conditions are fulfilled. So, we just have to let

$$
h^{11}=-\frac{1}{r} \int_{r_{0}}^{r}\left(r h^{1}+\frac{1}{r^{2}} \int_{r_{0}}^{r} \partial_{\theta}\left(-h^{2} r^{3}+\frac{r^{2}}{\cos \varphi} \partial_{\theta} h^{3}\right) d r+r \frac{\sin ^{2} \varphi}{\cos \varphi} h^{3}\right) d r .
$$

to conclude. 
Remark 2 If we suppose that the shell is subjected to a boundary condition of place along a portion its latteral face, for example on $r=r_{1}$ we have to add the following border conditions to $\mathbf{h}$ :

$$
h_{\mid \theta=\theta_{0}}^{2}=h_{\mid \theta=\theta_{1}}^{2}=h_{\mid \theta=\theta_{0}}^{3}=h_{\mid \theta=\theta_{1}}^{3}=\partial_{\theta} h_{\mid \theta=\theta_{0}}^{3}=\partial_{\theta} h_{\mid \theta=\theta_{1}}^{3}=0 .
$$

These equations are obtained by canceling the border integrals during the integration by parts.

\subsection{Parabolic shell partially clamped}

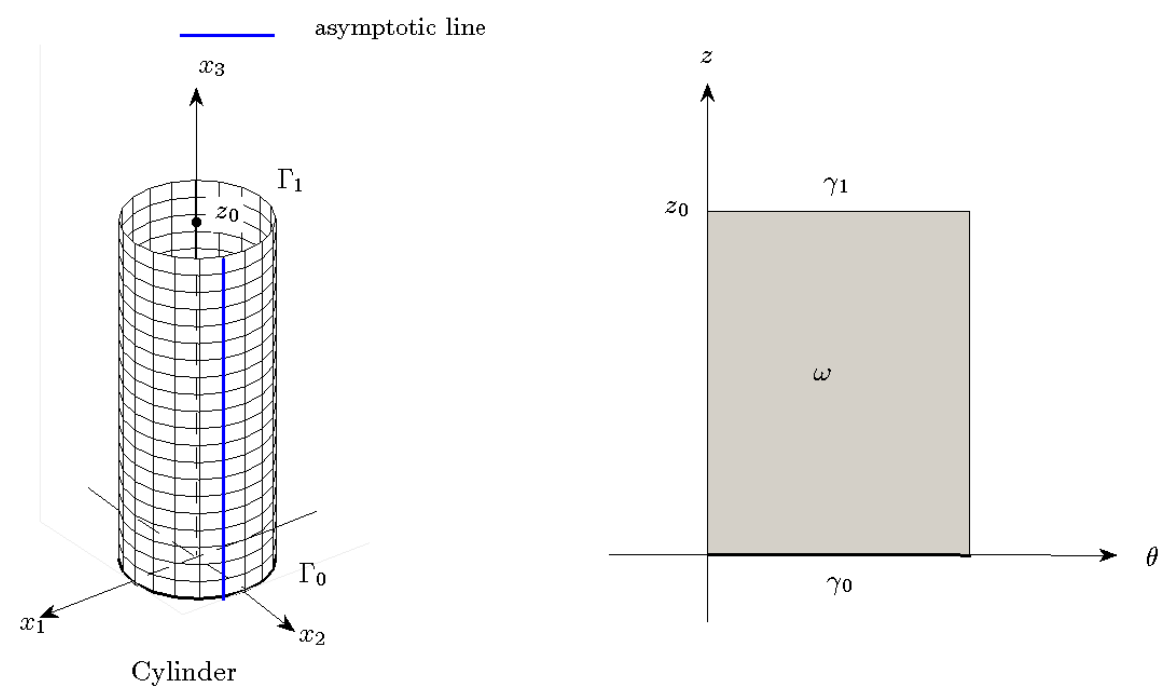

Figure 4: Parabolic shell partially clamped

Let us suppose that the middle surface of the shell is a portion of cylindar $\mathcal{C}$

$$
\mathcal{C}:=\left\{\left(x_{1}, x_{2}, x_{3}\right) \in \mathbb{R}^{3}, x_{1}=\cos \theta, x_{2}=\sin \theta, x_{3}=z \text { for }(\theta, z) \in \bar{\omega}\right\}
$$

where

$$
\omega:=] 0,2 \pi[\times] 0, z_{0}\left[\text { and } z_{0}>0,\right.
$$

and that the shell is subjected to a boundary condition of place along its entire "lower" face

$$
\Gamma_{0}:=\left\{\left(x_{1}, x_{2}, x_{3}\right) \in \mathbb{R}^{3}, x_{1}=\cos \theta, x_{2}=\sin \theta, x_{3}=0 \text { for } \theta \in[0,2 \pi[\} .\right.
$$

In these coordinates, the second fundamental form $\left(b_{\alpha \beta}\right)_{\alpha \beta}$ is

$$
b_{11}=-1, b_{12}=0 \text { and } b_{22}=0 \text {, }
$$

all the Christoffel symbols are equal to 0 whereas the jacobian $\sqrt{a}$ is equal to 1 . Let $\gamma_{0}:=\{(\theta, 0)$ for $\theta \in] 0,2 \pi[\}$ and $\gamma_{1}:=\left\{\left(\theta, z_{0}\right)\right.$ for $\left.\theta \in\right] 0,2 \pi[\}$. The displacement field is looked for in

$$
\mathbf{V}(\omega):=\left\{\mathbf{v} \in \mathbf{H}^{1}(\omega), \mathbf{v} 2 \pi \text {-periodic with respect to the first variable, } \mathbf{v}=\mathbf{0} \text { on } \gamma_{0}\right\} .
$$

The admissibilty condition (4) becomes : find $h^{\alpha \beta} \in L^{2}(\omega)$ such that for all $\eta=\left(\eta_{i}\right) \in \mathbf{V}(\omega)$

$$
\int_{\omega} h^{i} \eta_{i} d y=\int_{\omega}\left(h^{11}\left(\partial_{\theta} \eta_{1}+\eta_{3}\right)+h^{12}\left(\partial_{z} \eta_{1}+\partial_{\theta} \eta_{2}\right)+h^{22} \partial_{z} \eta_{2}\right) d y
$$


Theorem $\mathbf{7}$ The surface force $\mathbf{h}=\left(h^{i}\right) \in \mathbf{L}^{2}(\omega), 2 \pi$-periodic with respect to the first variable, is admissible if

$$
\partial_{\theta} h^{1}, \partial_{\theta} h^{3} \text { and } \partial_{\theta \theta} h^{3} \text { are in } L^{2}(\omega) .
$$

Proof. Let $\eta$ be in $V(\omega)$ and let $\mathbf{h}=\left(h^{i}\right)$ be a $2 \pi$-periodic with respect to the first variable function of $\mathbf{L}^{2}(\omega)$ satisfying (22). Taking successively as test function $\eta=(\eta, 0,0),(0, \eta, 0)$ and $(0,0, \eta)$ in (21) we obtain the three following equations satisfied by all $\eta \in V(\omega)$ :

$$
\begin{gathered}
\int_{\omega} h^{1} \eta d y=\int_{\omega}\left(h^{11} \partial_{\theta} \eta+h^{12} \partial_{z} \eta\right) d y \\
\int_{\omega} h^{2} \eta d y=\int_{\omega}\left(h^{12} \partial_{\theta} \eta+h^{22} \partial_{z} \eta\right) d y \\
\int_{\omega} h^{3} \eta d y=\int_{\omega} h^{11} \eta d y
\end{gathered}
$$

These equations are satisfied by

$$
h^{11}=h^{3}, h^{12}=\int_{z}^{z_{0}}\left(h^{1}+\partial_{\theta} h^{3}\right) d z \text { and } h^{22}=\int_{z}^{z_{0}}\left(h^{2}+\int_{z}^{z_{0}} \partial_{\theta}\left(h^{1}+\partial_{\theta} h^{3}\right) d z\right) d z .
$$

\section{Conclusion}

The method developped to obtain, from Theorem 2 and Theorem 3, sufficient admissibility conditions gives rather simple results (conditions of regularity and behaviour on the border). The difficulty to get these conditions depends on the geometry of the shell and on its clamping. For example, for elliptic partially clamped shells, it doesn't work. Nevertheless, Theorem 2 can be the start of other methods which lead to different sufficient admissibility conditions. One of them is developped in one example in Poutous [6].

\section{References}

[1] D. Chapelle and K. J. Bathe, The Finite Element Analysis of Shells Fundamentals, Computational Fluid and Solid Mechanics (Springer-Verlag Berlin Heidelberg, 2003).

[2] P. G.Ciarlet, Mathematical Elasticity, Vol. III. Theory of Shells, Studies in Mathematics and Its Applications, Vol. 29 (North-Holland Publishing Co., Amsterdam, 2000).

[3] P. G. Ciarlet and V. Lods, Asymptotic analysis of linearly elastic shells: "Generalized membrane shells", J. Elasticity 43(2) (1996) 147-188.

[4] V. Lods and C. Mardare, Justification des modèles linéaires de Koiter et de Naghdi pour des coques totalement encastrées soumises des forces "non admissibles", C. R. Acad. Sci. Paris, Ser I 333 (2001) 151-154.

[5] R. Luce, C. Poutous and J.-M. Thomas, Weakened conditions of admissibility of surface forces applied to linearly elastic membrane shells, C. R. Acad. Sci. Paris, Ser I 334 (2007) 721-726 .

[6] C. Poutous, Modélisation asymptotique et analyse numérique d'un problème de couplage fluidestructure, Thèse de doctorat, Université de Pau et des Pays de l'Adour, (2006).

[7] J. Sanchez-Hubert and E. Sanchez-Palencia, Coques élastiques minces. Propriétés asymptotiques, (Masson, Paris, 1997). 
[8] E. Sanchez-Palencia, On the membrane approximation for thin elastic shells in the hyperbolic case, Revista Matematica de la Universitad Complutense de Madrid 6(2) (1993) 311-331.

[9] S. Slicaru, On the ellipticity of the middle surface of a shell and its application to the asymptotic analysis of "membrane shells", J. Elasticity 46(1) (1997) 33-42 . 